\title{
Advanced quality control scanning system for electronic materials
}

\author{
Nathaly Castaneda ${ }^{1}$, Dhaivat J. Solanki ${ }^{2}$, James K. Meen ${ }^{2}$, Goran Majkic ${ }^{2}$ and Francisco C. Robles Hernandez ${ }^{2}$ \\ ${ }^{1}$ University of Houston, HOUSTON, Texas, United States, ${ }^{2}$ University of Houston, Houston, Texas, United \\ States
}

There is a current need for higher efficiency characterization for in-line materials systems. Raman spectroscopy and two-dimensional x-ray diffraction (2D-XRD) are powerful techniques to identify structural features from the micro to the nanoscale. However, they do not possess atomic resolution for further understanding of the accurate spatial and atomic distribution of the chemical makeup of electronic components or detailed chemical composition. Here we present a workflow proposal to generate a thorough characterization from the micro-scale to the atomic level. Figure 1 shows the typical characterization starting from Raman spectroscopy mapping methods, followed by 2D-XRD, focused ion beam (FIB), wavelength dispersion spectroscopy (WDS), and atomic resolution transmission electron microscopy (TEM).

Presented in Figure 1 is the workflow characterization example of $\mathrm{REBa}_{2} \mathrm{Cu}_{3} \mathrm{O}_{7-\mathrm{x}}(\mathrm{REBCO}$, rare-earth barium copper oxide) coated conductor (CC) films synthesized by advanced metal-organic chemical vapor deposition (A-MOCVD) system deposited on flexible Hastelloy/A12O3/IBAD-MgO/Homo-Epi-MgO/LaMnO3. Samples thickness $>4 \mu \mathrm{m}$ and composition of 50:50 mol.\% mixture of RE elements $(\mathrm{Gd}, \mathrm{Y})$ with an extra 30\% mol. RE content excess to form $\mathrm{RE}_{2} \mathrm{O}_{3}$ precipitates and introduction of $\mathrm{BaZrO}_{3}$ (BZO) nanorods by addition of a 15 mol.\% $\mathrm{Zr}$ were investigated in this study. The images are a simple representation of the work that is currently performed to assess and evaluate the potential reasons for the performance of superconductors. The method attempts to be helpful not only for superconductors but also for semiconductors and other electronic components.

Confocal micro-Raman spectrometer (XploRA, Horiba Jobin Yvon) equipped with 10x, 50x, and 100x objective lens, and three laser lines 532, 638, and $785 \mathrm{~nm}$, respectively, were utilized. The Raman system allows scanning from sub-micrometric to centimeter areas using an automated X-Y stage mode [1]. 2D-XRD was performed as a complementary monitoring tool for REBCO film characterization. Through this technique, the analysis and understanding of a wide range of defects such as heavy doping, texture, crystalline orientation, twins, among other features, are achievable [2]. The main inconvenience is the fact that it scans semi-large areas at a time. However, in combination with Raman spectroscopy, it makes a unique micro-submicron metric resolution scale. With Raman, it is feasible to identify the exact location where the major defective areas are as well as those with excellent habit characteristics as a function of active Raman frequencies, Raman shift or Raman intensity. This is where Focus Ion Beam (FIB) and the Wavelength-dispersive spectroscopy (WDS) become the tools of choice to extract and analyze critical areas for in-depth investigation. The FIB is operated from $30 \mathrm{kV}$ to $500 \mathrm{~V}$ for rapid extraction and then fine-tune polishing for the sample to reach a final thickness as thin as $4 \mathrm{~nm}$ or lengths within the wave extinction conditions. The intention is to have transparent samples to e-beam transmission with as low as possible interference with the potential to reveal atomic resolution images on STEM, TEM, EDS, WDS, EELS, HAADF, etc.

Acknowledgements

This work was supported by Advanced Manufacturing Institute, University of Houston, through award number 1121/H0579/B2001. We acknowledge University of Houston Division of Research High Priority Area Research Large Equipment Grant number 1026/H0073/B1004/I0503304 for establishing a state-of-the-art 2DXRD facility used in this study. The authors wish to express their gratitude to Thermo Fisher Scientific and the Nano Port-Portland team for their invaluable support with FIB and HRTEM (Dr. D. Gostovic, Dr. L. Pullan, Dr. L. Brockand, Dr. L. Casalena). 

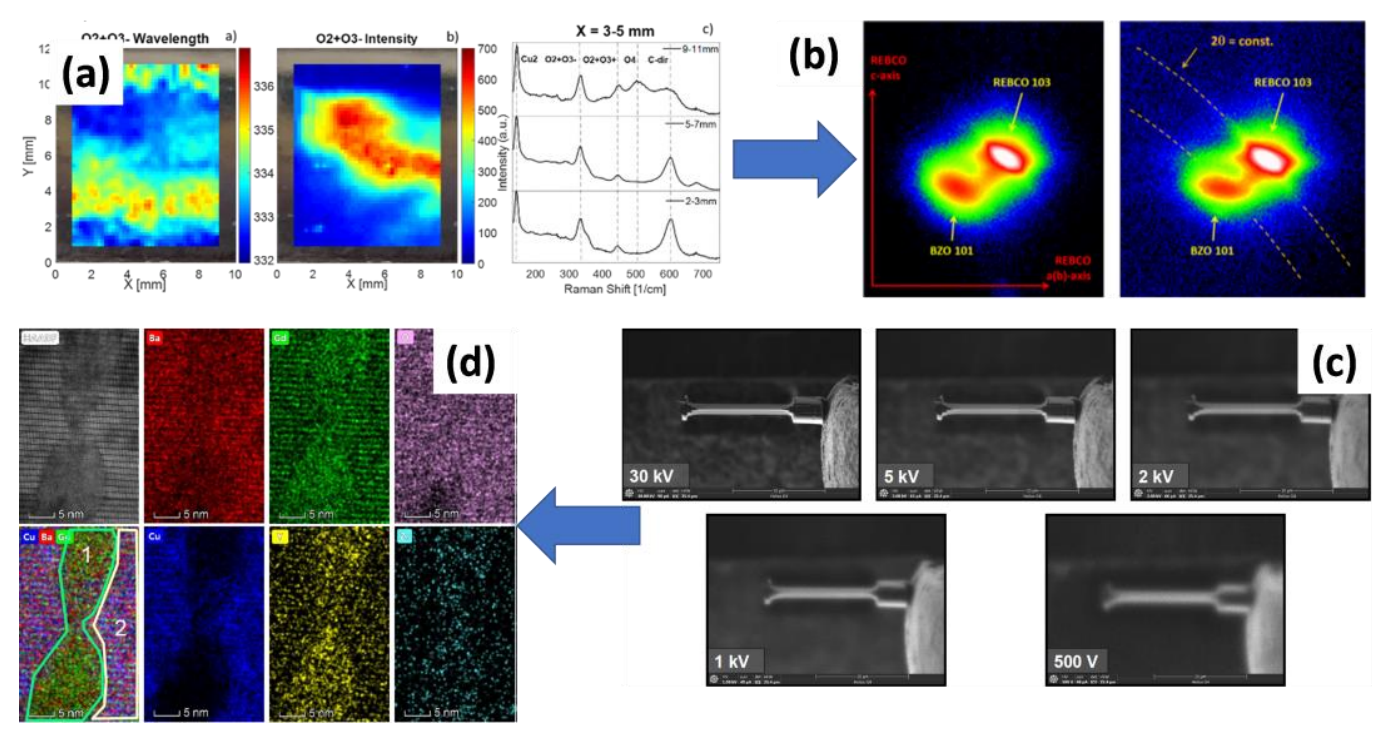

Figure 1. Workflow analysis from (a) microscopic (Raman) to (b) nanometric to (c) engineered sample preparation to $(\mathrm{d})$ atomic resolution.

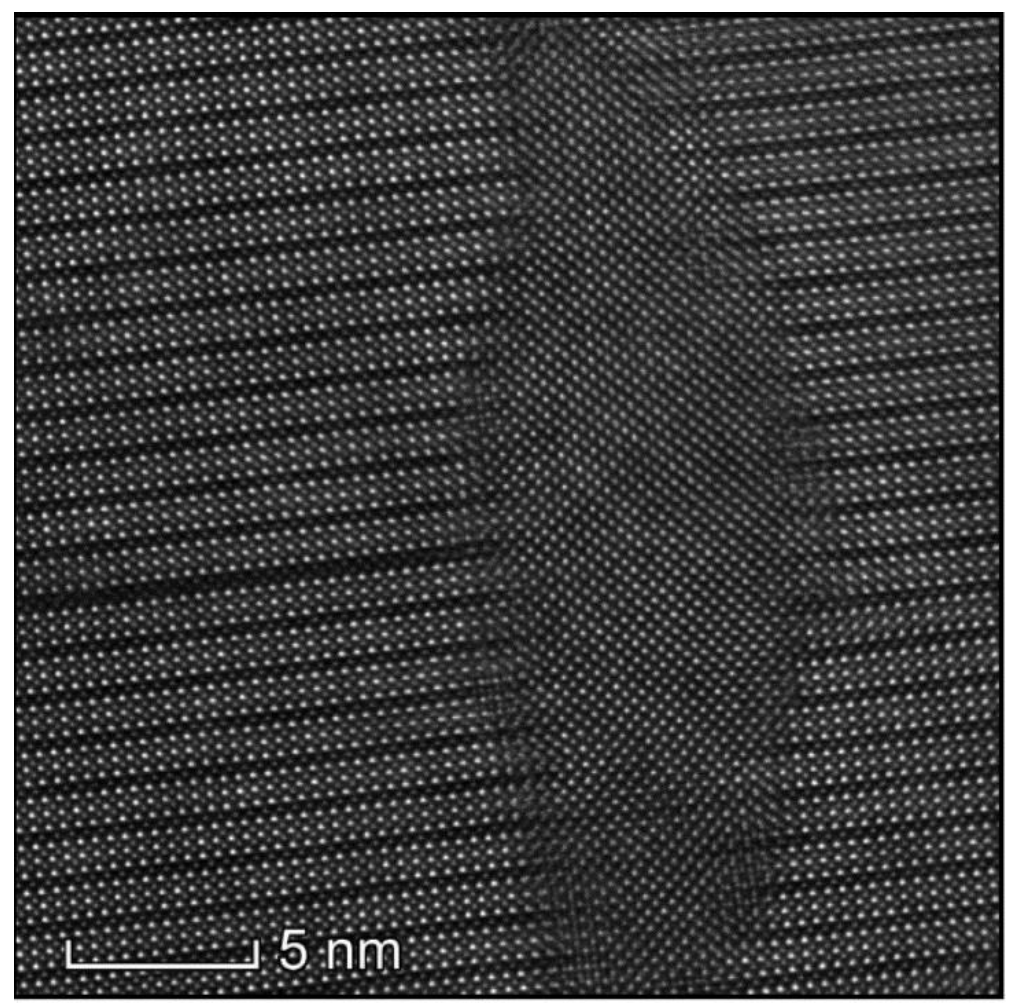

Figure 2. True atomic resolution of REBCO superconductor (matrix), showing a BZO nanorod.

\section{References}

1. Castaneda, N., G. Majkic, and F.C. Robles, Scanning Raman spectroscopy for inline characterization of 2G-HTS conductors. Superconductor Science and Technology, 2021. 34(3): p. 035032.

2. Majkic, G., et al., Engineering of Nanorods for Superior in Field Performance of 2G-HTS Conductor Utilizing Advanced MOCVD Reactor. Ieee Transactions on Applied Superconductivity, 2017. 27(4). 